\title{
Aberrant Multimeric Structure of von Willebrand Factor in a New Variant of von Willebrand's Disease (Type IIC)
}

\author{
Zaverio M. Ruggeri, Inga Marie Nilsson, Rossana lombardi, Lars Holmberg, \\ and Theodore S. Zimmerman, Angelo Bianchi Bonomi Hemophilia and \\ Thrombosis Center, Milan, Italy; Department for Coagulation Disorders, \\ Allmanna Sjukhuset, Malmo, Sweden; Departments of Immunology and \\ Basic and Clinical Research, Scripps Clinic and Research Foundation, La \\ Jolla, California 92037
}

\begin{abstract}
A B S T R A C T A variant of von Willebrand's disease has been identified in which sodium dodecyl sulfate agarose electrophoresis provides evidence that the von Willebrand factor present is structurally abnormal. Rather than the repeating triplet seen in normal subjects and in patients with the IIA and IIB variants, a repeating doublet was present in the propositus. None of the bands had the same mobility as bands in normal subjects or previously described von Willebrand's disease patients. The larger multimers of von Willebrand factor were lacking both from plasma and platelets, and did not appear in the circulation after infusion of 1-deamino-[8-D-arginine]-vasopressin. There was a marked increase in the concentration of the smallest multimer in the propositus and his phenotypically normal children, indicating that this abnormality of von Willebrand factor is inherited in an autosomal-recessive manner.
\end{abstract}

\section{INTRODUCTION}

Von Willebrand factor exists in plasma as a series of multimers extending in size from approximately

This is publication No. 2764 from the Immunology Department of Scripps Clinic and Research Foundation.

Address correspondence to Dr. Zimmerman, Scripps Clinic and Research Foundation, 10666 N. Torrey Pines Road, La Jolla, CA 92037.

Received for publication 7 July 1982 and in revised form 9 August 1982.
$M_{\mathrm{r}}=0.85 \times 10^{6}$ to over $M_{\mathrm{r}}=14 \times 10^{6}(1,2)$. However, these relative molecular weight values were calculated from relative mobilities in unreduced sodium dodecyl sulfate (SDS) agarose gels, and are likely to be overestimates of true relative molecular weight values $(3$, 4). The large multimers appear to be the most hemostatically important and are missing from the plasma of individuals with type II (variant) von Willebrand's disease $(1,5)$. We have recently shown that in normal plasma each multimer actually consists of a triplet represented by a predominant central band and two less prominent adjacent bands (4). In subtypes IIA and IIB von Willebrand's disease, the repeating unit is also a triplet and mobilities of all bands are the same as in normal subjects (4). In type IIA, however, the fastest migrating member of the triplet is increased in quantity out of proportion to the others (4).

We have now identified a new variant of von Willebrand's disease in which the repeating unit consists of only two bands, rather than three. In addition, neither band has the same mobility as those present in normal, IIA, or IIB triplets. Lastly, a marked increase in the concentration of the smallest multimer was present in the propositus and in his asymptomatic, presumably heterozygous, children. These findings provide evidence that the von Willebrand factor in this variant is structurally abnormal and that this abnormality is inherited in an autosomal-recessive fashion. 


\section{METHODS}

Patients and controls. The patients described in this study, as well as other normal volunteers from the Malmo and Milan Hospital staffs, were aware of the experimental nature of this study and gave their informed consent. The protocol for infusion of 1-deamino-[8-D-arginine]-vasopressin (DDAVP) ${ }^{1}$ was approved by the appropriate health authority of Sweden, where treatment was actually performed. The classification for the previously reported subtypes of von Willebrand's disease has been published $(5,6)$.

Procedures. Blood samples were collected by clean venipuncture and processed as described (6). Factor VIII procoagulant-activity, Factor VIII/von Willebrand factor-related antigen, ristocetin cofactor activity, and bleeding time were performed as previously published in detail (6).

The multimeric distribution of von Willebrand factor was analyzed in citrated plasma and platelet lysates by thin-layer agarose electrophoresis in the presence of SDS, using a discontinuous buffer system according to a method recently described (4). The bands corresponding to von Willebrand factor multimers were identified in the gels by reaction with ${ }^{125}$ I-labeled affinity-purified specific antibodies (emu antihuman von Willebrand factor) followed by autoradiography. No bands were identified in plasma from patients with severe von Willebrand's disease (4).

DDAVP was infused intravenously following a protocol previously described (6). Platelet lysates for analysis of the multimeric composition of platelet-associated von Willebrand factor were prepared as reported (1). Protease inhibitors were added before lysis, and lysates were electrophoresed within $1 \mathrm{~h}$ from preparation (1). Binding of plasma von Willebrand factor to washed platelets in the presence of ristocetin was assessed as previously described (7).

\section{RESULTS}

The propositus was a 39 -yr-old male, attending the Hemophilia Center in Malmo, Sweden, who had moderate bleeding symptoms and a markedly prolonged bleeding time. There was no family history of a bleeding diathesis. Both paternal and maternal families originated from the same isolated village in Sweden. The routine laboratory findings in the propositus and his children are summarized in Table $\mathbf{I}$.

Plasma was obtained from the propositus and his children on three separate occasions over a period of 6 mo and analyzed by SDS agarose gel electrophoresis using a discontinuous buffer system and $2.5 \%$ agarose (Figs. 1 and 2).

As with other type II variants of von Willebrand's disease, the larger multimers were absent from plasma, and, as in type IIA, were absent from platelets as well. However, the multimeric structure of the propositus' von Willebrand factor differed from that of normal as well as that of subtypes IIA and IIB von Willebrand's disease in several important respects. Most striking was the absence of the two bands adjacent to the central predominant band in each triplet (Fig. 1). In addition,

\footnotetext{
${ }^{1}$ Abbreviation used in this paper: DDAVP, 1-deamino[8-D-arginine]-vasopressin.
}

TABLE I

Clinical Expression and Routine Laboratory Data

\begin{tabular}{llrrrr}
\hline & $\begin{array}{c}\text { Bleeding } \\
\text { symptoms }\end{array}$ & $\begin{array}{c}\text { Ivy bleeding } \\
\text { time }\end{array}$ & VIIIC & VIIIR:Ag & VIIIR:RCo \\
\hline & & $m i n$ & & $U / d l$ & \\
Normal & - & $<9$ & $>50$ & $>50$ & $>50$ \\
Propositus (Male) & Moderate & $>30$ & 67 & 50 & 10 \\
Daughter & None & 9 & 95 & 95 & 60 \\
Son & None & 6 & 120 & 120 & 96 \\
\hline
\end{tabular}

VIIIC, Factor VIII procoagulant activity; VIIIR:Ag, Factor VIII/ von Willebrand factor-related antigen; VIIIR:RCo, ristocetin cofactor activity.

a new band was present in the space that normally intervenes between triplets. Thus, the repeating unit of the propositus' multimeric pattern contained two bands, instead of three. Examination of plasma from 50 normal subjects and from 80 patients with type $I$, IIA, and IIB von Willebrand's disease failed to disclose the intervening band seen in the propositus.

Two additional abnormalities of the propositus' multimeric structure were observed. First, the pre-

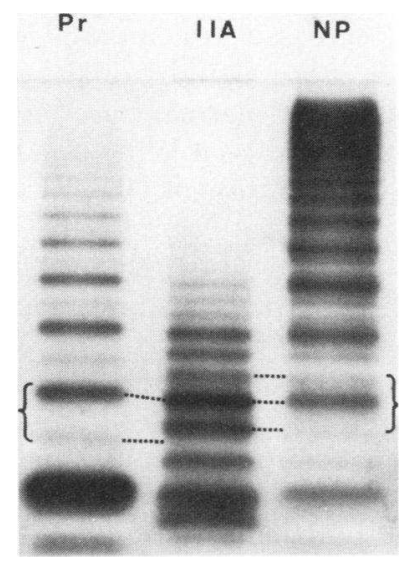

Figure 1 SDS $(0.1 \%)$ agarose $(2.5 \%)$ gel of von Willebrand factor in plasma of the propositus (Pr), an individual with IIA von Willebrand's disease (IIA), and a normal individual (NP). After electrophoresis, the gel was fixed in isopropanolacetic acid, reacted with ${ }^{125} \mathrm{I}$-affinity-purified anti-von Willebrand factor antibody, and an autoradiograph was made. The right-hand bracket includes the repeating triplet in both normal individuals and IIA von Willebrand's disease patients. The left-hand bracket indicates the repeating doublet in the propositus. It can be seen that bands with similar mobility are present in the plasma of normal individuals and IIA patients (connected by the lines between the middle and right-hand lanes), whereas the major repeating band in the propositus has a slower mobility than the major band in normal individuals and IIA patients, and the minor band in the propositus does not have an analogous band in normal individuals or IIA patients. The lettering has been placed at the bottom edge of the sample wells in the stacking gel. 


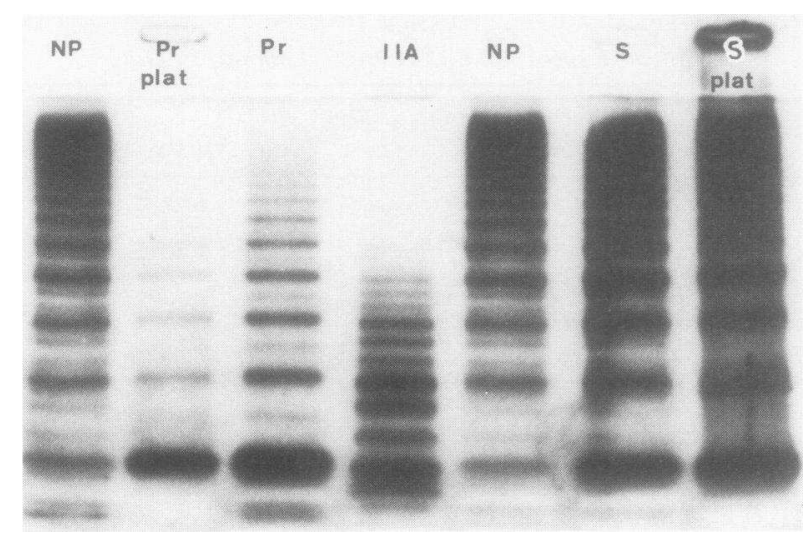

Figure 2 The entire SDS agarose gel from which Fig. 1 was taken. From left to right: plasma from a normal individual (NP), platelet extract from the propositus (Pr plat), plasma from the propositus (Pr), plasma from a patient with IIA von Willebrand's disease (IIA), an additional normal plasma (NP), plasma from the son (S), and platelet extract from the son ( $\mathrm{S}$ plat).

dominant band had a slightly slower migration rate than that of normal individuals or individuals with type IIA von Willebrand's disease (Fig. 1). In addition, the fastest moving multimer identified on agarose gels was markedly increased as compared with that from other variant or normal individuals. All the abnormalities displayed by plasma von Willebrand factor were present in platelet von Willebrand factor as well (Fig. 2), and administration of DDAVP did not change the abnormal plasma pattern.

The propositus' son and daughter had $(a)$ no bleeding symptoms, $(b)$ normal bleeding times, and $(c)$ normal measurements of Factor VIII/von Willebrand factor-related activities and antigens (see Table I). Moreover, SDS agarose electrophoresis showed that large multimers were present (Fig. 2). However, characteristic alterations of multimeric structure were also evident. The bands adjacent to the predominant triplet band were poorly defined (Fig. 2). More importantly, the fastest moving multimer showed the same disproportionate increase as was present in the propositus (Fig. 2).

The propositus' von Willebrand factor failed to bind to normal platelets in the presence of ristocetin, whereas normal binding was observed with von Willebrand factor from his two children.

\section{DISCUSSION}

Multimeric analysis of the von Willebrand factor in the propositus provided direct evidence of a structural abnormality. First, the characteristic repeating triplet present in normal von Willebrand factor is replaced with a doublet. Secondly, neither of the bands in the doublet has the same mobility as those in normal von Willebrand factor. These findings contrast with subtype IIB in which the triplet pattern is present, and with subtype IIA in which the triplet pattern is also present, though with a disproportionate increase in the concentration of the fastest migrating band (4). Peptide maps of IIA von Willebrand factor have failed to demonstrate a difference from normal von Willebrand factor (8).

Though extensive family studies were not possible, it is apparent that the mode of inheritance of this form of von Willebrand's disease differs from that of previously described variants. The family history on both paternal and maternal sides was completely negative for bleeding symptoms. This is often observed in the so-called autosomal-recessive form of severe von Willebrand's disease which, unlike this case, is characterized by extremely low levels of plasma and platelet von Willebrand factor (5). The son and the daughter of the propositus were clinically normal and although their von Willebrand factor possessed a normal complement of large multimers, abnormalities present on SDS agarose electrophoresis indicated that they were heterozygous for the defect displayed by their father. Thus, it is probable that the propositus was homozygous or doubly heterozygous for the genetic abnormality or abnormalities that gave rise to his structurally altered von Willebrand factor protein.

A previously reported family may have had the same abnormality as the one described here (9). However, only crossed immunoelectrophoresis was used to demonstrate the von Willebrand factor abnormality and therefore the multimeric structure could not be evaluated. Nevertheless, there was a distinct peak in the most rapidly migrating portion of the crossed immunoelectrophoresis pattern of the heterozygotes. This peak probably corresponds to the smallest multimer, which is so markedly increased in the heterozygotes reported here.

The propositus, like IIA and IIB patients, lacks the large multimers of von Willebrand factor in plasma. Therefore, we have chosen to designate this form of von Willebrand's disease as type IIC. Further investigation of the molecular abnormality present in this variant should provide new insights into the structure of von Willebrand factor and its genetic control.

\section{ACKNOWLEDGMENTS}

We thank Mr. James R. Roberts for excellent technical assistance and Ms. Roberta Novak-Buhrman for expert manuscript preparation.

This research was supported by grant CT 81.00347.04 from Consiglio Nazionale delle Ricerche, Rome, Italy; by grant RG 225.80 from the North Atlantic Treaty Organization; and by grant HL 15491 from the National Institutes of Health. 


\section{REFERENCES}

1. Ruggeri, Z. M., and T. S. Zimmerman. 1980. Variant von Willebrand's disease: characterization of two subtypes by analysis of multimeric composition of Factor VIII/von Willebrand factor in plasma and platelets. $J$. Clin. Invest. 65: 1318-1325.

2. Hoyer, L. W., and J. R. Shainoff. 1980. Factor VIIIrelated protein circulates in normal human plasma as high molecular weight multimers. Blood. 55: 1056-1059.

3. Counts, R. B., S. L. Paskell, and S. K. Elgee. 1978. Disulfide bonds and the quaternary structure of Factor VIII/von Willebrand factor. J. Clin. Invest. 62: 702709.

4. Ruggeri, Z. M., and T. S. Zimmerman. 1981. The complex multimeric composition of Factor VIII/von Willebrand factor. Blood. 57: 1140-1143.

5. Zimmerman, T. S., and Z. M. Ruggeri. 1982. von Willebrand's disease. In Progress in Hemostasis and Throm- bosis. T. H. Spaet, editor. Grune and Stratton, Inc., 6: 203-236.

6. Ruggeri, Z. M., P. M. Mannucci, A. B. Federici, R. Lombardi, and T. S. Zimmerman. 1982. Multimeric composition of Factor VIII/von Willebrand factor following administration of DDAVP: implications for pathophysiology and therapy of von Willebrand's disease subtypes. Blood. 59: 1272-1278.

7. Ruggeri, Z. M., F. I. Pareti, P. M. Mannucci, N. Ciavarella, and T. S. Zimmerman. 1980. Heightened interaction between platelets and Factor VIII/von Willebrand factor in a new subtype of von Willebrand's disease. N. Engl. J. Med., 302: 1047-1051.

8. Nachman, R. L., E. A. Jaffe, C. Miller, and W. T. Braun. 1980. Structural analysis of Factor VIII antigen in von Willebrand disease. Proc. Natl. Acad. Sci. USA. 77: $6832-6836$

9. Armitage, H., and C. R. Rizza. 1979. Two populations of Factor VIII-related antigen in a family with von Willebrand's disease. Br. J. Haematol. 41: 279-289. 